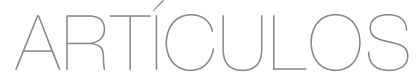

\title{
ORIGINALES
}

\section{Emprendimientos de la economía del conocimiento. Rol del financiamiento público en la provincia de Córdoba, Argentina*}

\author{
ANDRÉS COLOMBO** \\ MARÍA LORENA TALBOT-WRIGHT***
}

* Los autores quieren agradecer a la Universidad Siglo 21 por el financiamiento otorgado para la ejecución de este trabajo. ** Magíster en Administración de Empresas. Universidad Siglo 21, Córdoba, Argentina. E-mail: andres.colombo@ues21.edu.ar. ORCID: 0000-0002-4097-8164. Google Scholar: https://scholar.google.cl/citations?hl=es\&user=rG51KNAAAAJ.

*** Magíster en Propiedad Intelectual. Universidad Siglo 21, Córdoba, Argentina. E-mail: loretalbot@gmail.com. ORCID: 0000-0002-1216-0103. Google Scholar: https://scholar.google.cl/citations?hl=es\&user=qefj7GgAAAAJ. 


\section{COMO CITAR ESTE ARTÍCULO}

How to cite this article:

Colombo, A. y Talbot-Wright, M.L. (2021). Emprendimientos de la economía del conocimiento. Rol del financiamiento público en la provincia de Córdoba, Argentina. Revista Perspectiva Empresarial, $8(2), 65-76$.

Recibido: 28 de junio de 2021 Aceptado: 08 de octubre de 2021
RESUMEN Es indudable que la creación de nuevas empresas tecnológicas constituye uno de los motores del desarrollo. El rol del financiamiento público es clave para potenciar este tipo de emprendimientos; no obstante, el impacto de este tipo de financiamiento está poco estudiado. Objetivo. Analizar los emprendimientos apoyados por el Ministerio de Ciencia y Tecnología de la provincia de Córdoba (Argentina) a través de su programa FONTEC y medir el impacto del financiamiento público en estos emprendimientos. Metodología. Se analizaron 14 emprendimientos que ejecutaron FONTEC en 2018 y 2019, de distintas industrias, estudiando su estructura y gestión del financiamiento recibido. Resultados. Los resultados muestran que el principal problema asociado a este tipo de financiamiento fue la caída del poder de compra del dinero recibido dadas las condiciones macroeconómicas de Argentina en el período estudiado. Conclusiones. A pesar de sus dificultades, este tipo de financiamiento ha sido destacado como una de las pocas opciones de apoyo a la innovación en la región.

PALABRAS CLAVE economía del conocimiento, emprendedorismo, financiamiento, innovación, tecnología.

\section{Knowledge-economy ventures. The role of public financing in the province of Córdoba, Argentina}

ABSTRACT The creation of technological firms is unquestionable one of the engines of development. The role of public financing is critical in promoting this type of ventures; however, the impact of such funding has received little attention in the literature. Objective. To analyze the ventures in the province of Córdoba (Argentina) supported by the Ministry of Science and Technology through its FONTEC program and measure the impact of public funding on these ventures. Methodology. Fourteen ventures from various industries that participated in FONTEC in 2018 and 2019 were examined, with a focus on their structure and management of the funding received. Results. The results show that the major problem related to this type of financing was the decline in the purchasing power of the money received owing to Argentina's macroeconomic conditions during the study period. Conclusions. Despite its challenges, this type of financing has been highlighted as one of the few options for supporting innovation in the region.

KEYWORDS Knowledge economy, entrepreneurship, financing, innovation, technology. 


\section{As empresas da economia do conhecimento. 0 papel do financiamento público na província de Córdoba, Argentina}

RESUMO Não há dúvida de que a criação de novas empresas de tecnologia é um dos motores do desenvolvimento. 0 papel do financiamento público é fundamental para impulsionar este tipo de empreendimento; contudo, o impacto deste tipo de financiamento é pouco estudado. Objetivo. Analisar os empreendimentos apoiados pelo Ministério da Ciência e Tecnologia da província de Córdoba (Argentina) através do seu programa FONTEC e medir o impacto do financiamento público sobre esses empreendimentos. Metodologia. Foram analisados 14 empreendimentos que executaram o FONTEC em 2018 e 2019, de diferentes indústrias, estudando sua estrutura e gestão do financiamento recebido. Resultados. Os resultados mostram que o principal problema associado a este tipo de financiamento foi a queda no poder de compra do dinheiro recebido, dadas as condições macroeconômicas da Argentina no período estudado. Conclusões. Apesar das suas dificuldades, este tipo de financiamento tem sido destacado como uma das poucas opções para apoiar a inovação na região.

PALAVRAS CHAVE economia do conhecimento, empreendedorismo, financiamento, inovação, tecnologia. 


\section{Introducción}

Para alcanzar el desarrollo socioeconómico, el consenso general entre quienes definen y ejecutan políticas de innovación estipula que el conocimiento y el capital social son aspectos clave en el camino de países que buscan su transición hacia economías desarrolladas. Estos dos ingredientes de la fórmula del desarrollo han dado lugar al concepto de economía del conocimiento, considerada como una evolución de las fases de desarrollo posterior a la economía agrícola y a la economía industrial (Carayannis et al., 2006). La economía del conocimiento puede ser definida como producción y servicios basados en actividades intensivas en conocimiento que simultáneamente aceleran el avance técnico y científico y que producen una rápida obsolescencia, siendo el componente clave una mayor dependencia de las capacidades intelectuales que de los insumos físicos o los recursos naturales (Powell and Snellman, 2004).

En el contexto de una economía del conocimiento, los emprendedores tecnológicos tienen un rol crucial como catalizadores del desarrollo. Este tipo de emprendedores son considerados actores determinantes en el aprovechamiento de oportunidades comerciales a partir de la creación de soluciones intensivas en conocimiento (Muegge, 2013). En su impulso hacia la generación de empresas tecnológicas, el aporte de los emprendedores tecnológicos al desarrollo económico se vuelve tangible cuando se consideran los beneficios concretos relacionados con el establecimiento de más y mejores empresas de este tipo; las cuales redundan en la generación de nuevos empleos, la diversificación de la matriz productiva y un impacto directo sobre la dinamización del sistema de innovación (Talbot-Wright y Colombo, 2019). Si bien, entonces, resulta evidente el impacto positivo de los emprendedores en el desarrollo socioeconómico, en los países de América Latina las condiciones para la creación de emprendimientos intensivos en conocimiento reflejan la existencia de escaso capital humano emprendedor en un contexto marcado por un impacto relativamente bajo de la ciencia y la tecnología en el desarrollo de innovaciones y escaso acceso a financiamiento (Kantis, Federico e Ibarra, 2017).
¿Cómo se pueden favorecer contextos adecuados para la promoción de emprendimientos intensivos en conocimiento? Revisar este interrogante es el sentido de la mayor parte de las agendas de políticas para el desarrollo. Y en este marco la contribución del Estado como actor que estimula la innovación es un tema que ha sido largamente estudiado (Nelson, 1993; Peneder, 2008; Gnezdova et al., 2016; Reshetov et al., 2018; Gault, 2018; Dai, Li and Chen, 2021). Así, en el último tiempo, se ha resaltado su importancia radical en dinamizar la economía al estimular la innovación, sobre todo a partir del trabajo de Mazzucato (2013). Sin embargo existe aún mucha controversia respecto del alcance de tales políticas y el impacto que generan, en particular por la inexistencia de evidencia empírica específica sobre la contribución estatal (Torregrosa-Hetland et al., 2019).

La provincia de Córdoba, Argentina, muestra uno de los ecosistemas emprendedores más dinámicos del país. Particularmente hay un amplio tejido de instituciones de apoyo y fomento a los emprendedores tecnológicos con una participación activa por parte de agencias del Estado provincial en la promoción de emprendimientos innovadores. En este artículo se analiza el aporte del gobierno de la provincia de Córdoba al estimular la innovación a través de financiamiento público a nuevos emprendimientos tecnológicos mediante la iniciativa Fondo Tecnológico Córdoba - FONTECentre 2017-2019.

\section{Metodología}

En este trabajo se analizaron 14 emprendimientos de base tecnológica de la provincia de Córdoba, Argentina. Estos proyectos fueron apoyados por el FONTEC, que en el período 2017-2018 financió 58 proyectos de emprendedores.

La recogida de información de datos formales de los emprendimientos fue llevada a cabo a través del envío por correo electrónico y el análisis de la información colectada por parte del grupo de investigación. Se diseñó un cuestionario para indagar sobre los aspectos formales de los emprendimientos. Las respuestas se registraron 
a través de un formulario en línea, lo que permitió sistematizarlas de manera simple. Este formulario fue respondido por 14 emprendimientos de base tecnológica. Se trabajaron tres objetivos en la concepción del formulario: (i) su estructura contempló la adaptación de la información al lenguaje comúnmente utilizado por los encuestados; (ii) se buscó la simpleza en la formulación, minimizando el tedio y la fatiga; (iii) en relación con lo anterior, se buscó adaptar las preguntas de forma que sean claras para evitar confusión (Malhotra, 1997).

A partir de los resultados se procedió con la realización de entrevistas semiestructuradas a los emprendedores de los 14 proyectos. Se combinaron -en función a la disposición del encuestado y el contexto de aislamiento social, preventivo y obligatorio por la pandemia de la COVID-19llamadas por teléfono, videollamadas y audios. Se utilizó una guía para cada una de las entrevistas (figura 1). En este sentido la entrevista se diseñó partiendo de preguntas generales hacia preguntas específicas, introduciendo al encuestado en la temática. Se trabajaron principalmente preguntas en relación al equipo de trabajo, la estructura del emprendimiento, el análisis de intangibles, aspectos de mercado, modelo de negocios y recursos necesarios para el emprendimiento. Se transcribieron las entrevistas y luego se sistematizaron con base en la propuesta descrita por Huberman y Miles (1994) que indica la realización de pasos no secuenciales que permiten reducir la información, desplegar y luego extraer los datos. El principal objetivo en esta etapa fue trasladar el lenguaje oral al escrito para luego poder analizar y comparar la información. Se trabajó de manera iterativa, lo que permitió codificar y organizar de manera coherente la información para poder extraer las conclusiones.

\section{Gula de preguntas a emprendedores}

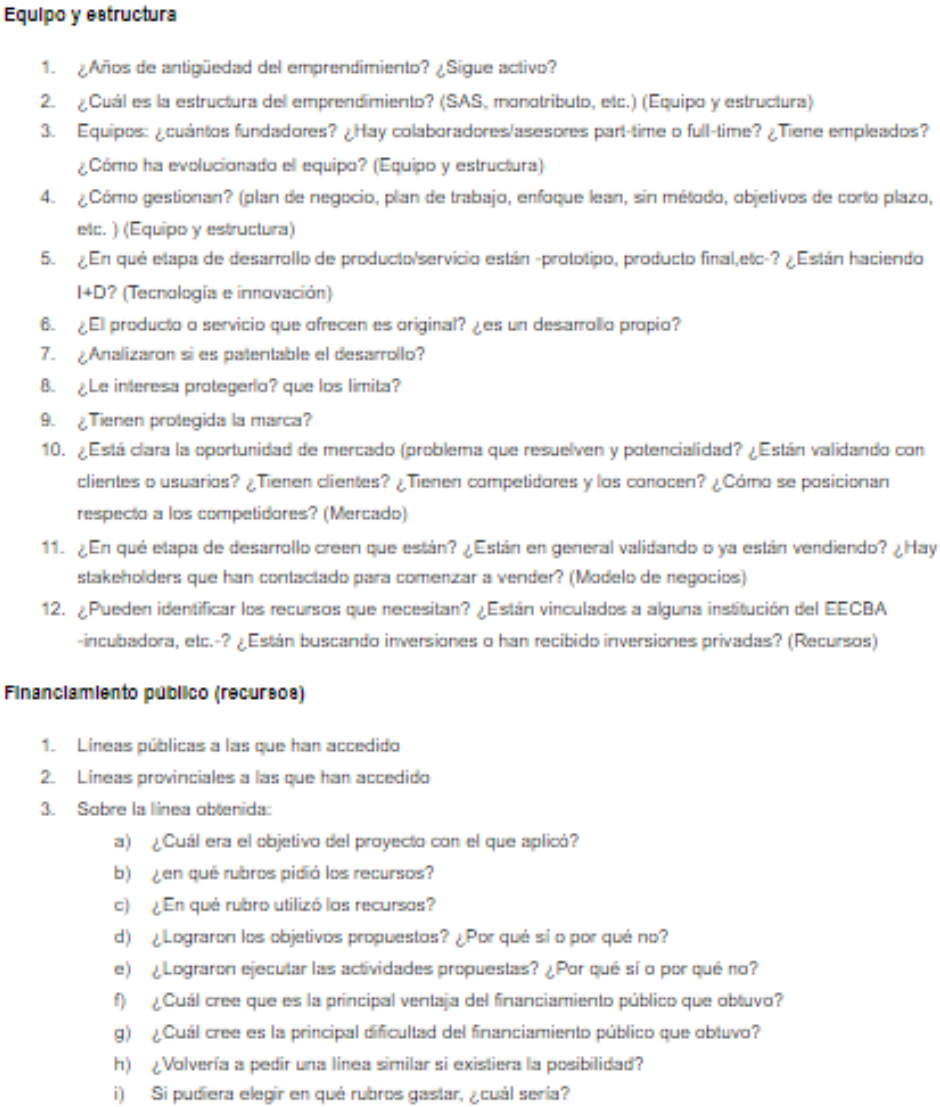

Figura 1. Guía de entrevista a emprendedores estudiados. Fuente: elaboración propia por parte de los autores. 


\section{Resultados y discusión}

El FONTEC es una línea de financiamiento del Ministerio de Ciencia y Tecnología - MinCyT_ de la Provincia de Córdoba que promueve el desarrollo de proyectos de media/alta intensidad científicotecnológica articulados entre el sector científico/ tecnológico y el sector productivo de la provincia. Esta línea ha tenido dos convocatorias a proyectos, en 2017 y 2018, poniendo a disposición de los emprendedores de la provincia fondos por un total de 10 millones de pesos argentinos en la edición 2017 y 17 millones de pesos en la edición 2018. La naturaleza de los proyectos financiados involucró iniciativas de diversos sectores productivos en los que se dio prioridad a áreas de biotecnología, nanotecnología, alimentos, mecatrónica, diseño industrial, tecnología de impresión 3D y microelectrónica, nuevos materiales, química fina, genética y genómica animal, vegetal y humana y tecnologías de la información y comunicaciones. En el alcance y los objetivos del FONTEC se destaca la intención por parte del MinCyT de financiar proyectos con una alta concentración de conocimientos científicos-tecnológicos orientados al (i) desarrollo de tecnología de media/alta intensidad a escala piloto, prototipo o laboratorio y (ii) producción de soluciones a problemas conducentes a la obtención de productos o procesos con una elevada integración de tecnologías de frontera. Cada proyecto seleccionado pudo acceder a un máximo de $\$ 400000$ en la convocatoria 2017 y a $\$ 450000$ en la convocatoria 2018 , representando este aporte un $70 \%$ del total del proyecto a ejecutar por cada postulante.

Además de los gastos en administración y ejecución del proyecto, el FONTEC financió gastos relacionados con: compra de equipamiento; insumos y/o materiales de construcción y/o adecuación de instalaciones. De igual manera los emprendedores pudieron financiar el desarrollo de las capacidades de sus emprendimientos tales como honorarios al personal de dirección técnica, investigación, contratación de expertos, asistencia y consultorías específicas. Asimismo, para emprendimientos con posibilidad de proteger sus desarrollos, FONTEC dispuso en sus convocatorias de un aporte extra de \$150000 para la protección a través de patentes. Los fondos también podían ser usados para la adquisición delicencias de tecnología, capacitacióny reentrenamiento de recursos humanos relacionados con las actividades del proyecto.

En estetrabajo seanalizaron 14 emprendimientos de un total de 58 proyectos aprobados, que accedieron a este financiamiento entre 2017-2018. Los emprendimientos estudiados fueron clasificados dentro de los sectores y áreas definidas por el FONTEC; la clasificación de los emprendimientos analizados se muestra en la figura 2.

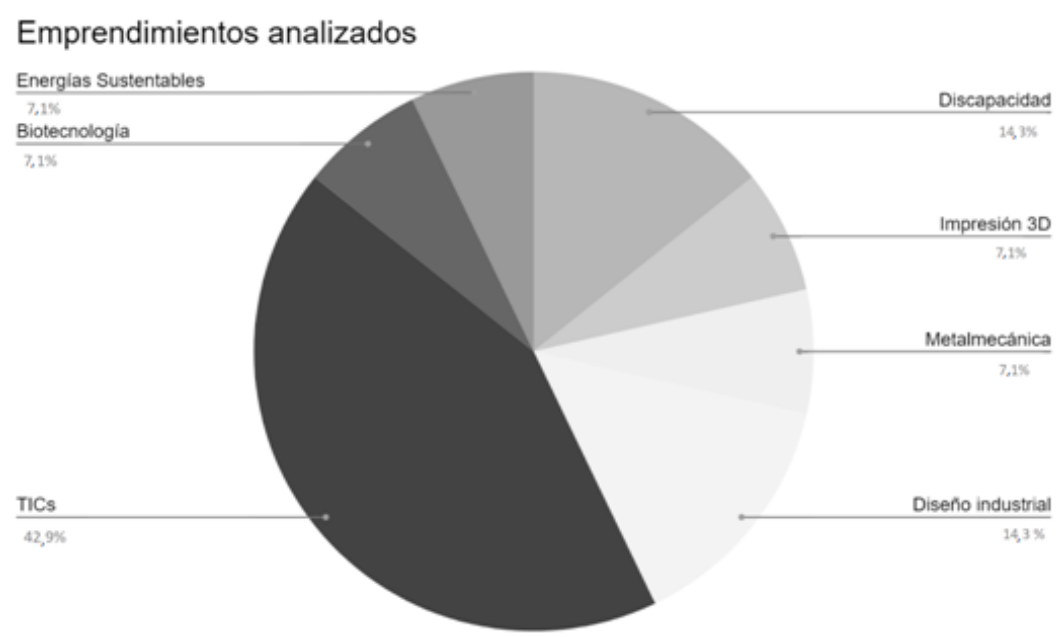

Figura 2. Clasificación de los emprendimientos analizados según los sectores productivos y áreas de aplicación priorizados por FONTEC. Fuente: elaboración propia por parte de los autores. 
La mayor parte de los emprendimientos estudiados corresponde a proyectos en el área de las tecnologías de la información y comunicaciones - TIC- Cabe destacar que el financiamiento FONTEC solo estuvo disponible para proyectos en esta área, orientados en forma excluyente a: (i) tecnología educativa; (ii) salud (procesamiento de imágenes, monitoreo en tiempo real, software y hardware); (iii) juegos infantiles para desarrollo de habilidades psicomotrices; (iv) robótica y domótica (software y hardware); (v) aplicaciones para gestión del conocimiento basadas en IA, machine learning y big data.

\section{Características principales de los emprendimientos analizados}

Hay una gama amplia de metodologías para gestionar emprendimientos, las cuales se encuadran bajo el concepto de métodos emprendedores (Entrepreneurial Methods). Muchos de estos pueden ser considerados como métodos prescriptivos, es decir, recetas sobre cómo los emprendedores deben razonar y actuar con el fin de generar un emprendimiento que cree valor (Mansoori and Lackéus, 2019). Sobre 14 emprendimientos analizados, 7 de ellos trabajan con metodologías que pueden ser categorizadas como tradicionales (esto es, métodos que se basan en planes de negocios y planes de trabajo). Este enfoque presupone que un emprendimiento es una versión pequeña de una empresa y que los resultados pasados pueden ser extrapolados hacia el futuro por el hecho de que no existe en un emprendimiento nuevo ninguna experiencia (McGrath, 2010; Blank and Dorf, 2012).

El resto de los emprendimientos se gestionaron con metodologías ágiles, que consisten en una gama de herramientas desarrolladas a partir de los trabajos de Steve Blank (Blank, 2007; Blank and Dorf, 2012; Blank, 2013); y con mayor impulso a partir de la irrupción del Lean Startup, una herramienta acuñada por Eric Ries, que se basa en la formulación y validación de hipótesis mediante la interacción de los emprendedores con sus clientes (Ries, 2011). En relación con este último enfoque, la mayoría de los emprendedores estudiados utiliza una mezcla de metodologías bajo esta categoría pero sin seguir de manera particular ninguna de ellas (entre las que mencionaron, se pueden destacar Lean Startup y Business Model Canvas).
En este sentido los 7 emprendimientos en este grupo trabajan bajo los conceptos generales de las metodologías ágiles, que pueden resumirse en la validación permanente de las hipótesis del modelo denegocios por parte de los emprendedores (Blank, 2013). Es interesante notar esta particularidad en la aplicación de metodologías ágiles por parte de los emprendedores entrevistados. Muchas veces se resalta la eficacia de las metodologías ágiles con base a su probada efectividad práctica; de hecho, el origen de esta filosofía de gestión está enraizado en la experiencia exitosa de emprendedores tecnológicos (en especial, en la industria de las TIC) sobre finales del siglo XX. El enfoque ágil dio origen a muchas herramientas en particular como las mencionadas - que consisten en más o menos pasos secuenciales y procedimientos de gestión detallados, aunque continúan siendo una prescripción para su adopción por parte de los emprendedores. Lo que siempre sobrevuela el ambiente emprendedor tecnológico es la capacidad de los equipos emprendedores de permanecer fieles a las metodologías de gestión y sus procedimientos particulares, especialmente en contextos cambiantes y en sectores industriales distintos a las TIC en los que las condiciones de mercado difieren (por caso, emprendimientos que desarrollan productos biotecnológicos con largos períodos de desarrollo tecnológico o en mercados de nicho).

Es así que los resultados obtenidos reflejan esta realidad, en el sentido de que los emprendedores entrevistados que gestionan con metodologías ágiles se apegan más a la filosofía o principios básicos de la metodología (validar para aprender rápidamente, utilizando prototipos o productos de mínimo valor - MVP-) que a herramientas y procedimientos particulares derivados de este enfoque.

Un concepto importante en el ámbito del emprendedorismo tecnológico es el de modelo de negocios. El modelo de negocios describe cómo una organización crea y distribuye valor, capturando una porción de este para poder ser sustentable económica y financieramente (Teece, 2010). En un emprendimiento, el modelo de negocios es dinámico: puede ser definido inicialmente por los emprendedores, pero la mayor parte del tiempo va cambiando y adaptándose en base a prueba y error (Yang, Sun and Zhao, 2019). Tan solo 5 de los 
emprendimientos analizados presentaron ventas recurrentes, un indicio de una mayor validación en el modelo de negocios. Un aspecto importante es que en este trabajo no se ha encontrado una correlación entre el nivel de ventas de estos emprendimientos y el método emprendedor utilizado para su gestión. Asimismo, un aspecto que resulta destacable es que los 5 emprendimientos con ventas recurrentes son los únicos que han logrado recibir inversión privada. Esta inversión se dio, en todos los casos, con posterioridad a la ejecución del financiamiento otorgado por FONTEC. Esta situación confirma la dinámica que el financiamiento privado a proyectos tecnológicos muestra en la provincia de Córdoba; en donde el proceso de inversión tiene un lugar fundamental en emprendimientos con ventas y clientes validados, algo que se da generalmente en la mayor parte de los ecosistemas.

La convocatoria FONTEC tenía previsto como gastos elegibles a aquellos relacionados con la protección de los desarrollos generados en el marco del proyecto; por ello dispuso un aporte extra de \$150000 para la protección a través de patentes. En una economía del conocimiento, los activos intangibles cobran especial interés en todo tipo de emprendimientos; en muchos casos, la importancia de este tipo de activos supera a la de los clásicos bienes tangibles. La preponderancia de los activos intangibles en emprendimientos tecnológicos se asocia con la posibilidad de transformarse en una fuente de ventaja competitiva que se puede identificar y valorizar (Suárez et al., 2020).

El $100 \%$ de los emprendimientos analizados considera que los productos o servicios que brindan son originales, creados a partir de procesos de investigación y desarrollo generados en el seno del emprendimiento. A su vez, el $64 \%$ de los emprendimientos mostró interés en proteger sus activos intangibles. En esta línea, el 29 \% de los emprendimientos entrevistados tienen solicitud de patente en trámite en Argentina; y remarcan que no solo enfocan esta estrategia para el monopolio de su desarrollo sino que también estiman que la protección de sus intangibles les permite posicionarse de mejor manera ante los inversores privados, quienes ven como positivo que un emprendimiento valore la protección de sus desarrollos.
Por su parte los emprendimientos que no muestran interés en la protección de sus intangibles asocian este desinterés con las características del sector industrial al que pertenecen, fundamentalmente la alta velocidad de desarrollo y actualización de los productos que no se adapta a los tiempos que conlleva tener una protección de derechos de propiedad intelectual. Más allá de la protección del know-how propio relacionado con el desarrollo de sus productos y servicios, el $86 \%$ de los emprendimientos tienen protegida su marca y valoran la necesidad de que el cliente relacione el signo marcario con el emprendimiento; lo anterior, lo utilizan como estrategia de marketing. Es necesario mencionar que la posibilidad de financiar la protección de intangibles a través de los fondos otorgados por el FONTEC resulta muy novedosa dado que ayuda a crear capital intangible a la empresa a través de la protección de sus conocimientos.

Cantnery Kösters (2015) determinaron que las empresas nacientessubvencionadas tienen 2,8 veces más patentes que empresas no subvencionadas y generan un $66 \%$ más de crecimiento del empleo. Por ello es de suma importancia mantener esta arista en el financiamiento para que estas empresas basen sus características innovadoras y mejoren su competitividad en un mercado potencial.

\section{La ejecución del financiamiento otorgado por FONTEC}

Las empresas emergentes -y de manera particular las empresas jóvenes- generan externalidades positivas que benefician su entorno y a la sociedad que las rodea, por lo que promueven la innovación. Estas empresas aplican a financiamiento público - tanto a créditos a tasas blandas como a subsidios - en busca de innovary poner en marcha su empresa, así como en algunos casos crecer. Según Hottenrott y Richstein (2020) proporcionar financiamiento público inicial a estas empresas reduce las fricciones en el mercado financiero y de esta manera el sector público compensa al emprendedor por los beneficios sociales que generan sus actividades como, por ejemplo, el aumento de empleo.

El financiamiento público otorgado a los emprendimientos tecnológicos tiene como destino distintos tipos de gastos e inversiones. Uno de 
los problemas en el financiamiento público tipo subsidio como es el del FONTEC radica en el tipo de gasto que deben realizar los emprendedores, denominados "gastos elegibles". Los recursos humanos son, en general, el principal insumo para el desarrollo de productos y servicios por los emprendedores tecnológicos en el área de las TIC. Tres de los emprendimientos estudiados y que desarrollan soluciones en este sector utilizaron la totalidad de los fondos para financiar la incorporación de recursos humanos bajo la forma de consultorías. Es importante mencionar que el FONTEC no permitió el financiamiento directo de recursos humanos propios sino su incorporación bajo la forma anterior. Este tipo de estrategias es común por parte de los emprendedores TIC, quienes constantemente mencionan la necesidad de líneas de apoyo que tengan la remuneración de recursos humanos como destino de sus fondos.

En Argentina, el fondo principal que ha permitido el financiamiento de recursos humanos por parte de proyectos TIC ha sido el Fondo Fiduciario de Promoción de la Industria del Software —FONSOFT—. Más allá de esto, es importante tener en cuenta que la postura predominante de quienes definen y gestionan el financiamiento público para innovación y emprendimientos tecnológicos es que sean los mismos emprendedores y/o ejecutores de los proyectos los que aporten los recursos humanos para su concreción. La mayor parte de los financiamientos disponibles permiten la inclusión de estos recursos propios de desarrollos como contrapartes en especies al proyecto, pues entonces no requiere el sacrificio de recursos monetarios por parte de los emprendedores sino su propia mano de obra aplicada a la ejecución del plan de trabajo planteado. Siguiendo este razonamiento, los proyectos que no cuenten con los recursos necesarios para el desarrollo de tal o cual tecnología específica pueden contratarlos mediante consultorías FONSOFT - que como se mencionó, sí son factibles de financiamiento por parte de la mayoría de las líneas, inclusive FONTEC-. De modo que, principalmente, las incompatibilidades aparecen cuando un grupo de emprendedores - con cierta expertise (por caso, el desarrollo de software)- intentan financiar con fondos públicos proyectos en los que el principal insumo es el recurso humano en áreas de su propio know-how (siguiendo con el ejemplo anterior, la creación de una plataforma Web). Es así que los emprendedores contratan como consultores a los mismos integrantes del equipo emprendedor, mecanismo que implementan como salvoconducto de las limitaciones anteriores. Como se mencionó, esta particularidad se dio en un porcentaje menor de proyectos. Así pues, los 11 proyectos restantes utilizaron los fondos disponibles fundamentalmente para la compra de insumos y equipos ( 6 de estos financiaron también servicios de consultorías en adición al rubro anterior).

En relación a la compleción de los proyectos presentados, tan solo el $50 \%$ de los emprendedores dice haber completado los objetivos iniciales propuestos para sus proyectos. Los 7 emprendimientos que no lograron completar de manera total la ejecución de lo planeado, y que solo cumplieron de manera parcial los objetivos, mencionaron como principal causa de ello el efecto de la inflación sobre los costos presupuestados. Durante 2018 (período en el que se ejecutaron los proyectos adjudicados en 2017), la inflación en Argentina fue del 47,6 \% (INDEC, 2019). Asimismo, la inflación de 2019 que afectó a los proyectos adjudicados en 2018 fue del 53,8 \% (INDEC, 2020). Esto repercutió de manera directa en la posibilidad de acceder a los recursos presupuestados por los emprendedores para la ejecución de su proyecto, ya que FONTEC no otorga fondos adicionales para adaptar el presupuesto de un proyecto adjudicado al aumento del precio de los recursos a incorporar por efecto de la inflación. Amén de lo comentado por los emprendedores que lograron una eficacia parcial en la compleción de sus objetivos, el 71 $\%$ han reconocido el efecto inflacionario sobre sus proyectos: más allá de haber logrado o no los objetivos planteados inicialmente, consideran la inflexibilidad del FONTEC para mantener el poder adquisitivo de los presupuestos como el aspecto más negativo de la línea de financiamiento. Este aspecto es crucial en la gestión de proyectos innovadores en Argentina.

Esta imposibilidad para adaptar los fondos otorgados a los incrementos en el nivel de precios se repite en la mayoría de las líneas de financiamientos públicos disponibles en el país. Al tener en cuenta los porcentajes importantes de inflación en los últimos años, el impacto negativo sobre los proyectos tecnológicos es rotundo; obligando a los emprendedores a modificar sus presupuestos y planes de trabajos, forzando decisiones de 
priorización que inevitablemente repercuten en los objetivos originales de los proyectos.

Por otro lado, la mitad de los emprendedores encuestados considera que las complicaciones burocráticas asociadas con la gestión de la línea FONTEC son otros de los aspectos negativos de esta línea. Incluidos en la gestión del financiamiento se encuentran actividades tales como la presentación del proyecto tecnológico que incluye su formulación y presentación de presupuestos, las actividades relacionadas con la gestión de cambios en presupuestos y actividades del proyectos una vez obtenido el financiamiento, la rendición de los gastos y el cierre técnico del proyecto ya ejecutado. Todos estos pasos de gestión de los fondos obtenidos implican generalmente un esfuerzo extra que los emprendedores no consideran al momento de buscar el financiamiento. Este hecho toma más relevancia cuando se trata de emprendedores tecnológicos que no cuentan en su estructura con recursos humanos con habilidades de administración (por ejemplo, relacionados con carreras afines a la administración de empresas). En estos casos, los emprendedores tecnológicos habituados a lidiar justamente con problemas de índole científicos y técnicos encuentran tortuosa la gestión administrativa del fondo obtenido.

Si bien es un paso inevitable, queda claro que la ponderación negativa de este aspecto está asociada a esta aversión para con las actividades de tipo administrativo que generalmente tienen aquellos que emprenden en tecnología. También es posible que existan complicaciones en la gestión y ejecución de los fondos que estén asociadas a problemas mismos de implementación por parte de las agencias de financiamiento, en este caso relacionadas con la estructura interna para llevar adelante el FONTEC por parte del MinCyT. Poder evaluar cuán efectivo es este organismo en la gestión de financiamientos, en especial del FONTEC, requeriría iniciar un estudio que abarque también la evaluación de otras reparticiones públicas nacionales que gestionan líneas similares; aspecto que no fue evaluado en este trabajo.

De acuerdo con Hottenrott y Richstein (2020) los gastos ejecutados en proyectos de innovación subvencionados se orientan principalmente a la adquisición de bienes tangibles no relacionados con proyectos de I +D, lo que no supone una probabilidad de innovación superior al de las empresas similares no beneficiarias de subsidios. A pesar de esto, hay un aspecto muy destacable y es que el $100 \%$ de los emprendedores estudiados considera que la principalventaja del FONTEC es su concepción como herramienta de financiamiento de investigación y desarrollo. Asimismo, y en consonancia con lo anterior, el $65 \%$ de los emprendedores reconoce que este tipo de líneas gestionadas desde el Estado son la única alternativa para el financiamiento de proyectos con alto contenido innovadory asociados a un mayor riesgo tecnológico.

Por último, 6 de cada 10 emprendedores mencionó que el principal cambio que debería sufrir el FONTEC es la posibilidad de incorporación del financiamiento de recursos humanos propios como uno de los destinos posibles de los fondos adjudicados. Tal como se discutió anteriormente, un aspecto crucial para la gestión de proyectos del área de las TIC se vuelve también relevante cuando hablamos de todos los sectores de la economía del conocimiento: es en estos emprendimientos tecnológicos en los que los recursos humanos son importantes e intrínsecos a los equipos emprendedores.

\section{Conclusiones}

No caben dudas que las opciones de desarrollo sostenible por parte de los países y sus regiones particulares deben incluir la generación de más y mejores empresas basadas en ciencia y tecnología. Fomentar y apoyar este tipo de empresas se vuelve entonces un tema central en la agenda de políticas de desarrollo. En este marco, poder estudiar el aporte que el Estado hace para la promoción de emprendimientos basados en ciencia y tecnología resulta particularmente relevante. Los resultados obtenidos en esta investigación muestran algunas particularidades relacionadas con la ejecución de este tipo de apoyo, en este caso en el contexto de un ecosistema a nivel regional dentro de un país en vías de desarrollo. Un análisis más profundo para el caso de una región como la provincia de Córdoba implica no solamente abordar una política en particular, sino también realizar un análisis del ecosistema emprendedor en el que se desarrollan los proyectos basados en ciencia y tecnología. 
En este sentido en una economía del conocimiento la innovación exitosa está asociada con la proximidad espacial tanto de los proveedores, clientes y centros de conocimiento (Audretsch et al., 2012; Guerrero et al., 2015; Fudickar y Hottenrott, 2018). Sin embargo analizar más casos e iniciativas como la propuesta en este trabajo, determinando el impacto del financiamiento público de distintos niveles sobre diversos tipos de emprendimientos, puede resultar una herramienta de discusión interesante para la generación de políticas públicas más efectivas que se traduzcan en la creación de empresas tecnológicas de impacto para una región.

\section{Referencias}

Audretsch, D.B. et al. (2012). Regional competitiveness, university spillovers, and entrepreneurial activity. Small Business Economics, 39(3), 587-601.

Blank, S.G. (2007). The four steps to the epiphany: successful strategies for products that win. San Mateo, USA: CafePress.

Blank, S.G. and Dorf, B. (2012). The startup owner's manual: the step-by-step guide for building a great company. Pescadero, USA: K \& S Ranch Publishing Llc.

Blank, S.G. (2013). Why the lean startup changes everything. Harvard Business Review, 5, 63-72.

Cantner, U. and Kösters, S. (2015). Public R\&D support for newly founded firms effects on patent activity and employment growth. Journal of Innovation Economics \& Management, 16, 7-37.

Carayannis, E.G. et al. (2006). Technological learning for entrepreneurial development (TL4ED) in the knowledge economy (KE): Case studies and lessons learned. Technovation, 26(4), 419-443.

Dai, X., Li, Y., and Chen, K. (2021). Direct demandpull and indirect certification effects of public procurement for innovation. Technovation, 101, 102198.
Fudickar, R. and Hottenrott, H. (2019). Public Research and the Innovation Performance of New Technology Based Firms. Journal of Technology Transfer, 44(2), 326-358.

Gault, F. (2018). Defining and measuring innovation in all sectors of the economy. Research Policy, 47(3), 617-622.

Gnezdova,J.V. etal. (2016). Conceptual model of the territorial manufacturing cooperative system use in Russia. Journal of Internet Banking and Commerce, 21(4), 82-94.

Guerrero, M. et. al. (2015). Economic impact of entrepreneurial universities' activities: An exploratory study of the United Kingdom. Research Policy, 44(3), 748-764.

Hottenrott, H. and Richstein, R. (2020). Start-up subsidies: Does the policy instrument matter? Research Policy, 49(1), 103888.

Huberman, M.A. y Miles, M.B. (1994). Manejo de datos y métodos de análisis. En Denman, C. y Haro, J.A. (Comps.), Por los rincones. Antología de métodos cualitativos de la investigación social (pp. 253-301). Hermosillo, México: El Colegio de Sonora.

INDEC. (2019). Índice de precios al consumidor (IPC). Informes Técnicos, 3(7), 1-9.

INDEC. (2020). Índice de precios al consumidor (IPC). Informes Técnicos, 4(5), 1-16.

Kantis, H., Federico, J. e Ibarra, S. (2017). Condiciones sistémicas para el emprendimiento dinámico 2017. América Latina: avances y retrocesos en perspectiva. Buenos Aires, Argentina: Universidad Nacional de General Sarmiento.

Malhotra, N.K. (1997). Investigación de mercados. Ciudad de México: Pearson Educación.

Mansoori, Y. and Lackéus, M. (2019). Comparing effectuation to discovery-driven planning, prescriptive entrepreneurship, business planning, lean startup, and design thinking. Small Business Economics, 54(3), 791-818. 
Mazzucato, M. (2013). The entrepreneurial state: Debunking public vs. private sector myths. London, England: Anthem Press.

McGrath, R.G. (2010). Business models: a discovery driven approach. Long Range Planning, 43(3), 247-261.

Muegge, S. (2013). Platforms, communities, and business ecosystems: Lessons learned about technology entrepreneurship in an interconnected world. Technology Innovation Management Review, 5-15.

Nelson, R. (1993). National Innovation Systems: A Comparative Analysis. Oxford, United Kingdom: Oxford University Press.

Peneder, M. (2008). The problem of private underinvestment in innovation: A policy mind map. Technovation, 28(8), 518-530.

Powell, W.W. and Snellman, K. (2004). The Knowledge Economy. Annual Review of Sociology, 30, 199-220.

Reshetov, K.Y. et al. (2018). State Policy in the Area of Implementation of Innovations in Industrial Production. The Journal of Social Sciences Research, 3, 271-276.

Ries, E. (2011). The lean startup: how today's entrepreneurs use continuous innovation to create radically successful businesses. New York, USA: Penguin Random House.

Suárez, J. et al. (2020). Valoración y auditoría de los activos intangibles. Una mirada conceptual. Revista Cubana de Contabilidad y Finanzas. COFIN HABANA, 0(1), 3-13.

Talbot-Wright, L. y Colombo, A. (2019). Propiedad intelectual y emprendimientos tecnológicos. Casos de emprendimientos de la ciudad de Córdoba, Argentina. Revista Perspectiva Empresarial, 6(1), 129-136.

Teece, D.J. (2010). Business models, business strategy and innovation. Long Range Planning, 43(3), 172-194.
Torregrosa-Hetland, S. etal.(2019). The prevalence of publicly stimulated innovations -A comparison of Finland and Sweden, 19702013. Research Policy, 48(6), 1373-1384.

Yang, X., Sun, S.L. and Zhao, X. (2019). Search and execution: examining the entrepreneurial cognitions behind the lean startup model.Small Business Economics, 52(3), 667-679. 